\title{
Advanced Tools for Clinical Diagnosis of Autism Spectrum Disorders
}

\author{
S. Orlandi ${ }^{1,2}$, C. Manfredi ${ }^{1}$, A. Guzzetta ${ }^{3}$, V. Belmonti ${ }^{3}$, S.D. Barbagallo ${ }^{1}$, and M.L. Scattoni ${ }^{4}$ \\ ${ }^{1}$ Dept. of Information Engineering, University of Firenze, Firenze, Italy \\ ${ }^{2}$ Dept. Electronic and Information Engineering "Guglielmo Marconi”, University of Bologna, Bologna, Italy \\ ${ }^{3}$ Dept. of Developmental Neuroscience, Stella Maris Scientific Institute, Pisa, Italy \\ ${ }^{4}$ Dept. of Cell Biology and Neurosciences, Istituto Superiore di Sanità, Roma, Italy
}

\begin{abstract}
Autism spectrum disorders (ASDs) are characterized by impairments in social and communication development and are not diagnosed before the second or the third year of life. Early identification of children with ASDs is a critical aspect for the management and treatment of this clinical condition. The aim of this study was the development of a tool for the improvement of the early diagnosis. This paper describes the design and development of this system, which overcomes screening performed with qualitative methods, integrating, in one software a basic patient data record, an audio/video data acquisition system, an editor for the creation of customized tests, a reporting system and an environment for test compilation. Finally, the system also provides interfaces allowing the analysis of the audio signals, such as infant crying, and the perceptual analysis of movements.
\end{abstract}

Keywords - Autism Spectrum Disorders, Acquisition System, Audio/Video Management and Analysis.

\section{INTRODUCTION}

Autism Spectrum Disorders (ASDs) are a group of complex disorders of brain development. These disorders are characterized, in varying degrees, by difficulties in social interaction, verbal and nonverbal communication and repetitive behaviors. They include autistic disorder, childhood disintegrative disorder, pervasive developmental disordernot otherwise specified and Asperger syndrome [1]. In most cases, ASDs symptoms appear in the first year of life, when it is not possible yet to carry out a reliable diagnosis. This is because the diagnostic tests currently used and considered gold-standard for ASD, are based on the identification of behavioral symptoms, which are more evident after the $24^{\text {th }}$ month of life. Moreover, etiology of ASDs is still unknown, although several studies identified abnormal neurotransmission in specific brain areas, such as limbic system, amygdala, cerebellum and sub cortical areas that mediate motor control [2].The study of the early symptoms of ASDs is mainly based on retrospective analysis of home videotapes, usually recorded during children's first birthday party[3], but this method presents some limits as. First of all, existing data refer to the assessment of a restricted number of infants (e.g. 10-12) [3, 4]. Moreover, only the pres- ence or absence of autistic behaviors was considered. Overall, the method may be not reliably standardized because of methodological differences in the quality of recording and in the setup of the observations. Recently, a prospective study of infant siblings of children with ASDs have been performed, using the Autism Observation Scale for Infants [5], which measures visual attention, response to name calling, social babbling, eye contact and sensory behaviors. Clinical research on newborn patient requires a noninvasive and marker-less technique that might aid in ASD early diagnosis, enabling a more effective intervention.

Recent studies support a strict relationship between cry and motor system development $[3,6]$. General movements (GMs) and crying could be considered early indicators of neurodevelopmental disorders, since they are both related to a functional neurotransmission in specific brain areas. These putative early indicators have been analyzed in several studies $[6,7]$. Specifically, cry involves activation of the central nervous system and requires a coordinated effort of several brain regions, mainly brainstem and limbic system. Preterm infants and infants with neurological conditions have different cry characteristics when compared to healthy infants $[8$, 9]. Being cheap and completely contact-less, cry analysis has recently aroused interest as an early sign of potential disturbances involving the central nervous system including ASDs [3]. Several studies [10,11] prove that in newborn infants affected by different brain lesions spontaneous motility loses elegance, fluency and complexity. Specifically, GMs, observable from the $9^{\text {th }}$ prenatal until the $21^{\text {st }}$ postnatal week, continue in a similar pattern until about the end of the second month post-term, followed by a gradually emerging new GMs patterns. Besides marker-based methods [10], in the last decades Prechtl's method was proven to be a highly sensitive and specific marker-less diagnostic tool for qualitative assessment of GMs, preventing discomfort the baby and without interfering with other measures saving time and costs [11]. Although the perceptual analysis of movements and crying has been already carried out in specialized clinics, the lack of automated tools for the objective diagnosis of ASD and the quality of its severity is highly required to save clinicians' time and reduce the margin of error. This is a timely challenge for health 
informatics and for biomedical engineering. In fact, the development of reliable software tools to enhance the early diagnosis $[12,13,14]$, especially acquiring patients data in real-life environments (i.e. home)[15, 16], has been highly investigated in the last years. One of the main needs emerged for these tools was the capability to enhance the diagnosis integrating clinicians' experience (mainly qualitative knowledge), with quantitative measurements [17, 18]. It is therefore important to develop semi-automatic qualitative methods to support the clinical diagnosis allowing for feature extraction and perceptual analysis with non-invasive marker-less technique.

This paper presents a new tool for the management of patient data, tests and reports, acquisition of audio and video data, their editing and analysis to support clinicians with evaluation of infant crying and general movements. Moreover, we strongly suggest assessment of these early indexes of infants' health during the first six months of age as their alterations could be considered early markers of Autism Spectrum Disorders.

\section{METHODS}

\section{A. Non-invasive Tool for Early Detection of ASDs}

This tool was developed with the support of the Italian grant project "Young Researcher 2008: Non-invasive tools for early detection of autism spectrum disorders", aiming to detect early markers of Autism Spectrum Disorders (ASDs) through the study of infant crying and GMs during the first six months of infant's life. This project aims to identify normative ranges for acoustical and motor parameters in a population of about 200 healthy newborn/infants, both male and female (control group). The control group will be compared with 15-20 "high-risk" newborn/infants, siblings of children already diagnosed with ASD [6, 7].

Infants were audio and video recorded at home five times during the first six months of life according to a specific protocol. The protocol includes also a clinical assessment performed using a set of questionnaires (Italian Questionnaire of Temperament; Bayley Scales of Infant Development; the first child vocabulary, MacArthur - Bates Communicative Development Inventory and the Modified Checklist for Autism in Toddlers (M-CHAT). Informed consent has been obtained from parents. The study protocol was approved by the local ethical committee (Istituto Superiore di Sanità, Roma, Stella Maris Hospital, Pisa and Bambin Gesù Hospital, Roma, Italy).

Cry analysis was carried out by the estimation of acoustic parameters such as fundamental frequency (F0), intensity, resonance frequencies of the vocal tract, length of each cry episode and crying melody (F0 shape). GMs analysis was performed with a perceptual technique that is often used by clinicians to diagnose motors problems associated with impairment of the central nervous system such as the early diagnosis of cerebral palsy [11].

\section{B. ARAD System}

According to the project [6] and in co-operation with ISER Tech srl (Prato, Italy), we developed a new tool for audio/video acquisition and analysis for contact-less diagnosis, in particular in neonatology area. The ARAD system (Acquisition, Reporting and Analysis for Diagnosis), shown in fig. 1, allows the management of personal data and medical history of the patient, data acquisition, personalized test editing, audio/video editing and reporting in a single software tool.

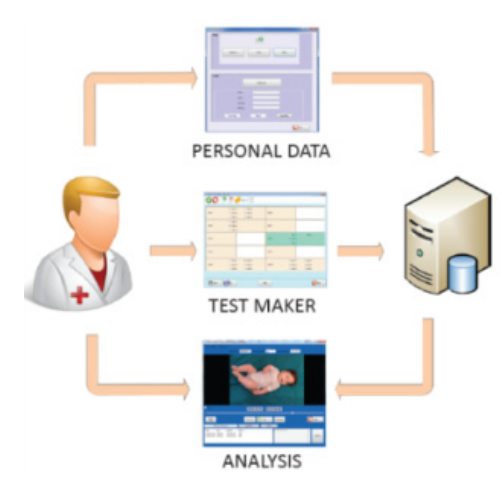

Fig. 1: ARAD System.

The system was developed in $\mathrm{C \#}$ language using the OpenCv image processing library for video acquisition and recording. It consists of four parts:

a) Personal Data Management System

an integrated management system to share data and medical history with dedicated interface and for patient's clinical storyboard. It allows importing and exporting signals, images and tests from external sources.

b) Test Maker Tool

an interactive environment for the creation and run of specific clinical tests with modular customizable structure. It is possible to insert multimedia contents, images, audio/video screenshots and monitor the results.

c) Recording Tool

an integrated environment for capturing multichannel audio/video with the possibility to enter notes, and contextualized information through the wizard and flexible setup of the system.

d) Analyzer System

tool devoted to the perceptual analysis and audio/video editing. Provides the ability to easily cut/copy/evaluate sequences of interest and enter clinical assessments without the need to resort to the use of other software. 
Patient data records are managed through a centralized database structured to guarantee privacy and personal data protection [6].

\section{ARAD for Acquisition and Reporting}

The acquisition system (fig. 2) is designed to be used also from patient's home to minimize the discomfort for the involved subjects, and to minimize biases due to external environment on children habits. The system was tested with different webcams or cameras with USB and different microphones. The ARAD system allows the user to enter most of the data from medical records. Automatic importing from already existing medical records is possible but it depends on the system used in the hospital. In any case, the user can enter the required historical data for this type of analysis.

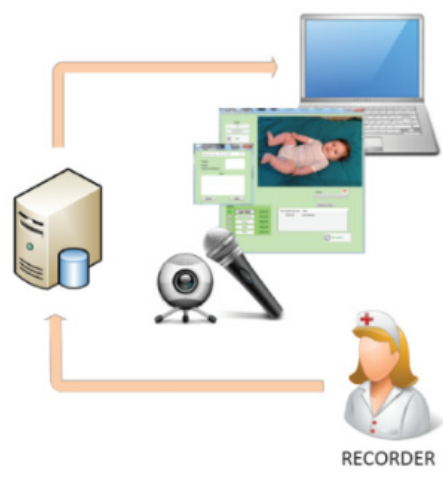

Fig. 2: Acquisition Tool.

Once the user has entered the patient's data, he can proceed through the special section for the audio/video recording. The user interface includes a main window for selecting the devices for the recording and an acquisition window.

The acquisition window allows previewing video and audio quality before starting the recording, in order to ensure the quality of the acquisitions. This tool allows also: marking the time frames corresponding to the different behavioral states of the newborn; showing separate running times for the crying state to assess the duration of the audio signal available for the crying analysis as well as the calm awake state required for the GMs analysis. The user can also select other markers that identify the state of the patient such as sleep. He can enter notes while recording as well as markers for ambient noise or interfering events to speed up the analysis. It is also possible to make multiple recordings on the same patient. During recordings it is possible to fill test and questionnaires imported or built with ARAD test maker. The special reporting system has been tested by several clinicians and it was considered user friendly, fast and intuitive, allowing them to have everything organized for the clinical evaluation.

\section{ARAD for Analysis and Diagnosis}

ARAD is equipped with specific tool for the analysis of audio and video recordings. It allows facilitating the actions carried out by the clinician during the perceptual studies. ARAD can achieve two different analyses, one for the sound, especially for crying and one for video recordings, in particular for GMs:

\section{a) Cry analysis}

After the identification of the crying frames, it is possible to perform time-frequency analysis. The extracted parameters, according to the methods used in $[6,7,8,9$, 19] are: the fundamental frequency of cry excerpts (F0) and the resonance frequencies, the number of cryepisodes, the vocal percentage, the number and the length of the voice breaks.

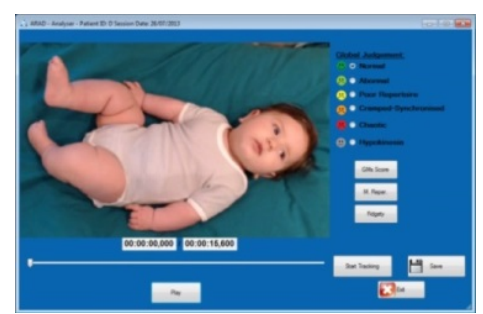

Fig. 3: ARAD Interface for perceptual movements analysis.

\section{b) General movements analysis}

The clinician can built specific tests without the need of pen and paper, when the part of video that contains the GMs has been selected by means of the "test maker" option. The tests, imported or inserted through test maker, can be compiled and exported. It is possible to exchange this information via email with other authorized clinicians. It is also possible to enter information about the number of motion sequences, the number of abnormal movements, the duration and the time at which they occurred.

Finally, the user can eventually view the patient's report and carry out the diagnosis, through the storyboard and the medical data.

This advanced tool differs from existing video/audio editing software because it allows, through a simple interface, to select the parts of interest, using markers placed during the recordings process and permits clinicians to enter comments and notes on single crying or motion sequences, necessary for the perceptual analysis $[10,11]$. Moreover, the system permits to carry out recordings both at the subject's home and in the hospital, such as in neonatal intensive care units. The system has been equipped with two different access levels: a restricted one for nurses or paramedics for the data acquisition and an unrestricted one for doctors who can perform the crying and general movements analyses. 


\section{RESULTS AND CONCLUSIONS}

To our knowledge, the system described in the present study is the first tool for recording and analyzing both cry and general movements in infants. The core modules have been individually tested $[6,8,9]$ by our group: audio and video files have been analyzed by clinicians (perceptual analysis) and their results correlated with automated analysis obtained by ARAD system. The whole system is undergoing testing in the clinical centers now and will undergo a specific assessment according to ISO standards for medical devices and for human centered tool design. The ARAD system has some limitations due to the environmental settings and the quality of recordings that could interfere with the analysis accuracy. ARAD is a tool that supports the clinical and perceptual evaluation but it is not meant to replace them. At present, only two systems are available for cry acquisition and analysis, in particular for the study of F0, but no tool exists for the analysis of GMs $[8,9]$. As most of recent studies $[3,4,5,7]$ highlight the possibility of recognize some typical signs of autism in the first year of life ARAD could give a significant support to early diagnosis of this disorder. In addition to providing appreciable decrease in investigation time, costs and errors, ARAD meets the growing clinical interest for marker-less monitoring for clinical data analysis and diagnosis. With few modifications, the tool could be used for the screening of a wide range of peri-and post-natal pathologies and can be easily adapted for homecare of infants.

\section{ACKNOWLEDGMENT}

Supported by the Italian Ministry of Health Grant (GR3), Young Researcher 2008, "Non-invasive tools for early detection of Autism Spectrum Disorders". The authors thank ISER Tech srl and Dr Leandro Pecchia for his external contribution to this work.

\section{CONFLICT OF INTEREST}

The authors declare that they have no conflict of interest.

\section{REFERENCES}

1. APA (2000). Diagnostic and statistical manual of mental disorders (4th ed., text rev.). Washington, DC: APA.

2. Bauman ML, Kemper TL, (2005) Neuroanatomic observations of the brain in autism: a review and future directions. Int J DevNeurosci. 23(2,3):183-187

3. Esposito G,Nakazawa J,Venuti P,Bornstein MH (2013) Componential deconstruction of infant distress vocalizations via treebased models: A study of cry in autism spectrum disorder and typical development, ResDevDisabil, 34(9): 2717-2724

4. Baranek GT (1999) Autism During Infancy: A Retrospective Video Analysis of Sensory-Motor and Social Behaviors at 9-12
Months of Age, J Autism Dev Disord,29(3):213-224

5. Bryson SE, Zwaigenbaum L, McDermott C et al. (2008) The Autism Observation Scale for Infants: scale development and reliability data, J Autism Dev Disord, 38(4): 731-738

6. Bocchi L, Orlandi S, Manfredi C, Puopolo M, Guzzetta A, Vicari S, Scattoni ML (2011) Early Diagnosis of Autism Spectrum Disorders Design of the Data Acquisition and Management System, 5th European Conf. of the Int.Fed. Med. Biol.Eng, Budapest, Hungary, IFMBE Proc.37: 187-190

7. Orlandi S, Manfredi C, Bocchi L,Scattoni ML (2012) Automatic newborn cry analysis: a non-invasive tool to help autism early diagnosis, ConfProc IEEE Eng Med BiolSoc,2012:2953-2956

8. Manfredi C, Bocchi L, Orlandi S, Spaccaterra L, Donzelli GP (2009), High-resolution cry analysis in preterm newborn infants, Med. Eng. \&Phys, 31(5):528-532

9. Orlandi S, Bocchi L, Donzelli GP, Manfredi C (2012) Central blood oxygen saturation vs crying in preterm newborns, Biomed Signal Proces, 7(1):88-92

10. Einspieler C, Prechtl HFR (2005) Prechtl's assessment of general movements: A diagnostic tool for the functional assessment of the young nervous system, MentRetard DevDisabil Res Rev.11(1):61-67

11. Einspieler C, Prechtl HFR, Bos AF, Ferrari F, and Cioni G (2004) Prechtl's Method on the Qualitative Assessment of General Movements in Preterm, Term and Young Infants. Cambridge University Press

12. Melillo P, Bracale M, De Luca N, Pecchia L (2013) Classification tree for risk assessment in patients suffering from Congestive Heart Failure via long-term Heart Rate Variability, IEEE J Biomed Health Inform, DOI: 10.1109/JBHI.2013.2244902

13. Melillo P, Izzo R, De Luca N, and Pecchia L (2012) Heart Rate Variability and Target Organ Damage in Hypertensive Patients, BMC CardiovascDisord, 12:105

14. Melillo P, Pecchia L,Testa F, Rossi S, Bennett J, Simonelli F (2012) Pupillometric analysis for assessment of gene therapy in Leber Congenital Amaurosis patients, BiomedEng Online, 11(1): 40

15. Melillo P,Bracale M,Pecchia L(2011) Nonlinear Heart Rate Variability features for real-life stress detection. Casestudy:students under stress due to university examination, Biomedical Engineering Online 10. doi:Artn 96 Doi 10.1186/1475-925x-10-96

16. Pecchia L, Melillo P, Bracale M (2011) Remote Health Monitoring of Heart Failure With Data Mining via CART Method on HRV Features, IEEE Trans Biomed Eng, 58 (3):800-804.

17. Pecchia L, Martin JL, Money AG, Barnet J (2013) Application of Analytic Hierarchy Process for user needs elicitation. A preliminary study on a device for auto-injection of epinephrine, HIS2013, London, UK. In G. Huang et al. (Eds.): HIS 2013, LNCS 7798, pp. 258264. Springer, Heidelberg

18. Pecchia L, Martin JL, Ragozzino A, Vanzanella C, Scognamiglio A, Mirarchi L, Morgan S (2013) User needs elicitation via analytic hierarchy process (AHP).A case study on a CT scanner, BMC Med Inform DecisMak, 3(1):2

19. Manfredi C (2000) Adaptive noise energy estimation in pathological speech signals, IEEE Trans Biomed Eng,47(11):1538-43

Author: Silvia Orlandi

Institute: Dept. of Information Engineering

Street: Via S. Marta, 3

City: Firenze

Country: Italy

Email: silvia.orlandi@unifi. 